\title{
Application of PCR-based DNA sequencing technique for the detection of Leptospira in peripheral blood of septicemia patients
}

\author{
Shylaja Ram, ${ }^{1}$ Jeyalatha Mani Vimalin, ${ }^{1}$ Malathi Jambulingam, ${ }^{1}$ Vaidehi Tiru, ${ }^{2}$ Ravi Kumar Gopalakrishnan ${ }^{3}$ and \\ Madhavan Hajib Naraharirao. ${ }^{1 *}$ \\ ${ }^{1}$ L \& T Microbiology Research Center, Vision Research Foundation, Sankara Nethralaya, 18, \\ College Road, Chennai- 600 006. India. \\ ${ }^{2}$ Clinical Microbiology Laboratory, Sundaram Medical Foundation, $873,17^{\text {th }}$ Main Road, \\ Shanthi Colony, 4th Avenue, Anna Nagar, Chennai -600040, India. \\ ${ }^{3}$ Leptospirosis research laboratory, Madhavaram Milk Colony, Chennai-600051. \\ E-mail:drhnm@snmail.org
}

Received 2 May 2011; received in revised form 18 October 2011; accepted 14 December 2011

\begin{abstract}
Aim: Isolation, dark field detection and microscopic agglutination test (MAT) are considered "gold standard" tests for diagnosis of Leptospirosis. Several PCR assays are reported but very few have been evaluated for detection of Leptospirosis. Therefore, this study was undertaken. This study aims to design and standardize polymerase chain reaction (PCR) -based DNA sequencing technique for the detection of pathogenic Leptospira from peripheral blood of patients clinically diagnosed with septicemia. Methodology and Results: Two hundred and seven (207) blood samples from patients were diagnosed with septicemia which includes 100 bacterial (other than Leptospira) culture positive and 107 bacterial culture negative samples were studied. Primers for Nested PCR targeting LipL32 gene of Leptospira interrogans were designed and the specificity of primers was tested against serum samples positive/negative by either MAT or dark field microscopy. PCR amplified products were further confirmed by DNA sequencing. The standardized nPCR was sensitive and specific to Leptospira interrogans. Twenty-one (21\%) out of 100 culture positive blood samples, three $(2.8 \%)$ out of 107 culture negative samples showed nPCR positivity and were confirmed as Leptospira interrogans by DNA sequencing $(p<0.001)$. A sensitive nPCR specific to Leptospira interrogans was developed. Conclusion, significance and impact of study: The $p$ value $(<0.001)$ signifies that Leptospira is commonly associated with other bacteria circulating in blood indicating that a decreased immune status is created primarily by a bacterium with enhanced possibility of development of Leptospiral infection probably be of an endogenous origin.
\end{abstract}

Keywords: DNA sequencing - Leptospira - LipL32 gene -polymerase chain reaction.

\section{INTRODUCTION}

Leptospirosis is a widespread zoonotic disease in humans caused by Leptospira. The clinical spectrum of the infection ranges from sub clinical to severe illness with high mortality rate. Human Leptospirosis causes severe multi organ dysfunction that may end in multi organ failure and death (Suganthan and Varghese, 2005). Leptospira interrogans is acquired through contact with animal reservoirs or an environment contaminated by their urine. Although it is considered to be the most geographically widespread zoonosis, it has traditionally been a sporadic disease of rural and tropical settings. It is restricted to high-risk groups such as farmers, miners, abattoir and sewer workers, and those who involved in recreational activities (Urimala et al., 2002). Outbreaks have raised new awareness concerning the risk of Leptospirosis in disaster situations (Center for disease prevention, 1998, 2000) and sporting (Pan American Health Organisation, 1998 and Sanders et al., 1999) events. Exposure to infected water in a canal was also reported as cause of the Leptospirosis outbreak (Jena et al., 2004). Another study describe the seroepidemiologic and clinical characteristics of the leptospirosis outbreak in 2008 in Sri Lanka (Agampodi et al., 2011) .

More than 200 Leptospira interrogans serovars have been identified as pathogenic and over 60 as nonpathogenic, which are Leptospira biflexa (Joythi et al., 2004). They are indistinguishable morphologically or by growth characteristics (Joythi et al., 2004). It has long been known that Leptospires spreads almost immediately from the site of entry of skin or mucous membrane, via lymphatics to the blood stream, where they circulate to all tissues (Faine, 1994) However, only $30 \%$ of Leptospirosis patients are correctly diagnosed (Chu et al., 1998).

Failure to recognize acute Leptospirosis means a delay in the initiation of its therapy and possibly ensuing severe complications such as Weil's syndrome. If the host survives the acute infection, septicemia and multiplication of organism persist until the development of opsonizing immunoglobulin in plasma, followed by rapid immune clearance. However, after clearance from blood,

\section{*Corresponding author}


Leptospires remain in immunologically privileged sites, including renal tubules, brain and anterior chamber of the eye, for weeks to months (http://emedicine.medscape.com/article/220563-overview). Recently, co-infection of Leptospira with other organisms has been reported. Leptospiral co-infection with malarial parasite, (Chansuda et al., 2003) dengue virus,(Rele et al., 2001) scrub typhus (Yuhawaiho et al., 2006) and Salmonella (Osbold et al., 2008 , Ronsholt et al., 2009) have been reported in the previous studies. Diagnosis of Leptospirosis is usually accomplished by serology, because culture requires both special media and incubation for several weeks. Molecular methods have recently been attempted as an alternative to culture (Marinelli et al., 2007 and Hernandez et al., 2011). PCR is found to be the most simple, specific and rapid method for detection as well as differentiation of Leptospira as pathogenic or non-pathogenic when compared to clinical methods. Many other methods like the recombinant antigen-coated latex beads could detect the specific antileptospiral antibodies in the acute phase of the illness have been proposed in recent years (Dey et al., 2007). The test is simple and inexpensive, and is rapid in the management of large numbers of patients. Several PCRbased methods targeting various regions have been developed recently for the detection of Leptospira in clinical specimens (Suganthan and Varghese, 2005). In this study, we designed and standardized a nested PCR (nPCR) for Leptospira interrogans targeting the gene for the major outer membrane lipoprotein LipL32, which appears to be an important virulent factor confined to pathogenic strains of all Leptospira spp. (Merien et al., 1992; Gravekamp et al.,1993; Noubade et al., 2002). Several lines of evidence indicate that LipL32 is a lipoprotein modified in a manner similar to that of other prokaryotic lipoproteins. LipL32 is also a prominent immunogen during human Leptospirosis, sequence and expression of LipL32 is highly conserved among pathogenic Leptospira species (Fonseca et al., 2009). As this gene is specific to pathogenic Leptospira, nested PCR technique was standardized and applied onto peripheral blood specimens collected from patients diagnosed with septicemia.

\section{MATERIALS AND METHODS}

\section{Study population}

Two hundred and seven peripheral blood samples from 207 consecutive patients admitted for septicaemia with clinical symptoms of high fever, chills, hypotension, and body pain, vomiting at Sundaram Medical Foundation, Chennai, Tamil Nadu, and South India. During the period September 2008 to August 2009 were included in this study. Children, patients with prolonged antibiotics, catheterized patients, patients undergoing intubations were excluded. The study was approved by the Research and Ethics subcommittee of both the institutes and the patients were informed of the possible use of blood samples for scientific purpose. Informed consent was recorded in writing in the patient's file as required by the Ethical Committees.

\section{Specimen collection}

Peripheral blood was collected after disinfecting the area of collection with $70 \%$ alcohol. The blood was directly collected in blood culture bottle (BacT/ALERT ${ }^{\circledR}$ BIOMERIX). In addition five hundred microliters of blood was collected at the same time for $\mathrm{nPCR}$ and isolation of Leptospira. Blood samples were stored at $-80^{\circ} \mathrm{C}$ until further processed.

One hundred samples were tested positive for culture by BacT/ALERT®BIOMERI UX Durham, USA (carried out at Sundaram Medical Foundation) and the other 107 samples were tested negative. The bacteria isolated in BacT/ALERT®BIOMERIUX, Durham, USA were further identified by Mini-API system. The results of blood cultures and nPCR were kept masked between the two groups of investigators and were comparatively analyzed only at the end of study period.

\section{Cultivation of Leptospira from blood specimens}

Two reference strains were obtained from Leptospira Diagnostic Centre, TANUVAS, Madhavaram, Tamil Nadu, were used in the study. The reference strains included were Leptospira interrogans serovar australis, Leptospira interrogans serovar autumnalis. These were maintained in Elling Hausen- Mccullogh- Johnson-Harris (Difco,BD Biosciences, USA) medium in the laboratory.

Attempts at isolation of Leptospira were made only with specimens tested positive for Leptospira DNA. Inocula consisting of 10 drops of blood stored at $-80^{\circ} \mathrm{C}$ was inoculated in EllingHausen-Mccullogh-Johnson-Harris ((Difco,BD Biosciences, USA) medium enriched with leptospira enrichment solution (Difco,BD Biosciences, USA). Ten $\mu \mathrm{g} / \mathrm{mL}$ 5-fluouracil (5-FU ${ }^{\circledR}$, Sigma, USA) was incorporated in the medium to avoid contamination. Cultures were incubated at room temperature (approximately $24^{\circ} \mathrm{C}$ ). The tubes were checked for growth every week. If there was no growth at the end of three months the cultures were considered negative for Leptospira.

\section{Molecular Tests}

Standardization of nested Polymerase Chain Reaction

\section{(i) DNA extraction}

DNA was extracted from $200 \mu \mathrm{L}$ of EDTA anti coagulated whole blood samples and reference strains using QIAAMP blood DNA extraction kit (QIAGEN, Hildan, Germany). The DNA extraction was carried out according to the manufacturer's instruction. The DNA was quantified using Nanodrop DNA quantifier - (Nd-1000. spectrophotometer, version 3.3, Wellington - USA.) and stored at $-80^{\circ} \mathrm{C}$ until further processed. 


\section{(ii) Primer designing and PCR standardization}

Primers for nested PCR targeting LipL32 gene were designed by using primegen and primer premier 5 software (Palo Alto CA, USA). Primers for round 1 resulting in a 322 bp product was designed by using primegen and the primers for round 2 resulting in $98 \mathrm{bp}$ product within the $322 \mathrm{bp}$ region were designed using primer premier 5 software. Two sets of primers with the following sequences were chosen and used for the PCR optimization. Round I:

LipL32 F1-5'CCCAGGGACAAACGAAAC3',

LipL32R1-5'TTTGGTCAGGCATAATCG3',

Round II:

LipL32F25'GGACGGTTTAGTCGATGGAA3',

LipL32R25'CCTGTTGGGGAAATCATACG 3'.

The PCR reaction mixture consisted of a $50 \mu \mathrm{L}$ reaction volume, containing $5 \mu \mathrm{L}$ template DNA, $5 \mu \mathrm{L}$ of $10 \mathrm{X}$ reaction buffer with $15 \mathrm{mM} \mathrm{MgCl}_{2}, 100 \mathrm{mM}$ of dNTP mixture, $1 \mu \mathrm{L}$ of $1 \mu \mathrm{M}$ forward, $1 \mu \mathrm{L}$ of $1 \mu \mathrm{M}$ reverse primers and $3 \mathrm{U} / \mu \mathrm{L}$ of Taq DNA polymerase. All PCR reagents were procured from Banglore Genei Pvt Ltd, Bangalore, India.

The amplification profile consists of a total of (PerkinElmer 3700, Applied Biosystems, USA) 35 cycles of $94^{\circ} \mathrm{C}$ for $1 \mathrm{~min}, 60^{\circ} \mathrm{C}$ for $1 \mathrm{~min}$, and $72^{\circ} \mathrm{C}$ for $1 \mathrm{~min}$ with initial denaturation at $95{ }^{\circ} \mathrm{C}$ for $5 \mathrm{~min}$ and final extension at 72 ${ }^{\circ} \mathrm{C}$ for $7 \mathrm{~min}$. Round II was carried out in the same way as round I except the annealing was maintained at $57^{\circ} \mathrm{C}$. One microliter of the amplified product from the round I was transferred to round II.

Two reagent controls, one consisting of reagents, first round primers and water alone, another specific for the second round (consisting of PCR reagents, water, and second round primers) and a known positive control with DNA extracts of Leptospira interrogans standard strains added were included in each PCR run. PCR products were visualized in $4 \%$ Agarose gel with VILBER Lourmet Gel Documentation System, France. Results were considered to be valid only when negative control (reagent control) of the II and I round PCR showed no band and the positive control showed a $98 \mathrm{bp}$ product at the end of II round. For clinical specimens, presence of $98 \mathrm{bp}$ band at the end of II round was considered positive. DNA extraction, setting up of $\mathrm{PCR}$ reaction for amplification and post-PCR handling was performed in physically separated rooms. Separate pipettes and barrier tips were used to prevent carry over contamination. Filter guarded tips and dedicated pipettes were used to prevent cross over and aerosol contamination.

Sensitivity of the PCR was estimated using serial 10fold dilutions of the DNA extracted from standard strain. Sensitivity was repeated twice to ensure the consistency. In vitro analytical specificity of the PCR was checked against human DNA and various bacterial, fungal, viral DNA which includes Staphylococcus aureus ATCC 6538, Bacillus subtlis lab isolate, Escherichia coli ATCC 4157, Pseudomonas aeruginosa ATCC 9742, Klebsiella pneumoniae lab isolate, Mycobacterium tuberculosis H37 RV, Proteus vulgaris lab isolate, Haemophilus influenza ATCC16211, Acinetobacter ATCC 9956, Salmonella typhi lab isolate, Enterobacter aerogenes lab isolate, Citrobacter koseri lab isolate, Candida albicans ATCC90028, Herpes Simplex Virus- 1 ATCC 733-VR.

The laboratory isolates against which the analytical specificity was tested were identified using standard biochemical reactions and wherever the results of standard biochemical reactions were inconclusive, PCR-based DNA sequencing techniques was applied for identification. The percentage difference between specimens positive only for Leptospira PCR and specimens' positive for bacterial culture as well as Leptospira nPCR was statistically analyzed by application of Chi-square test.

\section{(iii) Validation of the Primers}

Specificity of the primers was evaluated against the "gold standard", Microscopic agglutination test (MAT) by performing $\mathrm{nPCR}$ against 25 serum samples that showed the presence of antibodies to Leptospira by MAT. PCR was also carried out with 20 more serum samples which were tested leptospira positive by dark field microscopy and 5 other samples tested negative. Twenty five MAT negative serum samples were also included. The MAT tested serum samples and serum samples tested by dark field microscopy were obtained from Leptospira Diagnosstic Centre, Tamil Nadu University of Veterinary and Animal Sciences (TANUVAS), Madhavaram. In addition a serum sample collected from a patient with clinical symptoms of jaundice, neuro leptospirosis, acute renal failure who was MAT positive, IgM ELISA (40 PANBIO units) positive was also included.

\section{(iv) Testing the PCR products by DNA sequencing}

The amplified products in the samples positive by $\mathrm{nPCR}$ were subjected to DNA sequencing. Cycle sequencing of the amplified products was performed in a $10 \mu \mathrm{L}$ reaction volume, containing $0.5 \mu \mathrm{L}$ of $\mathrm{RR}$ mix, 3.5 $\mu \mathrm{L}$ of sequencing buffer, $0.5 \mu \mathrm{L}$ of forward primer $(1: 100$ diluted), $3.5 \mu \mathrm{L}$ MilliQ water and $0.5 \mu \mathrm{L}$ of amplified product. Amplification was carried out in Perkin-Elmer thermocycler (Applied Biosystems,USA) using 25 cycles of $96{ }^{\circ} \mathrm{C}$ for $10 \mathrm{~s}, 50^{\circ} \mathrm{C}$ for $5 \mathrm{~s}, 60^{\circ} \mathrm{C}$ for 4 min with initial denaturation at $96{ }^{\circ} \mathrm{C}$ for $1 \mathrm{~min}$. The cycle-sequenced products were then purified and sequenced using $\mathrm{ABI}$ Prism 3130 AVANT (Applied Biosystems, USA) genetic analyzer that works based on the principle of Sangers' dideoxy termination method. The sequences were then analysed by Bio Edit sequence alignment software, and BLAST analysis [www.ncbi.nlm.nih.gov/BLAST] was done to confirm the sequenced data with the standard strains and to determine the percentage homology.

\section{RESULTS}

Two hundred and seven peripheral blood samples from 207 patients with septicemia were taken. The age of the patients ranged from 20 - 80 years, and the mean age was 44.3 . The male to female ratio was equal to 1 . 
DNA extraction from the standard Leptospiral culture and clinical specimens by QIA-AMP blood DNA extraction kit yielded a good amount of DNA without any protein contamination. The primers were sensitive enough to detect a DNA concentration of $1 \mathrm{fg}$ and it specifically amplified Leptospiral DNA (Figures 1 and 2).

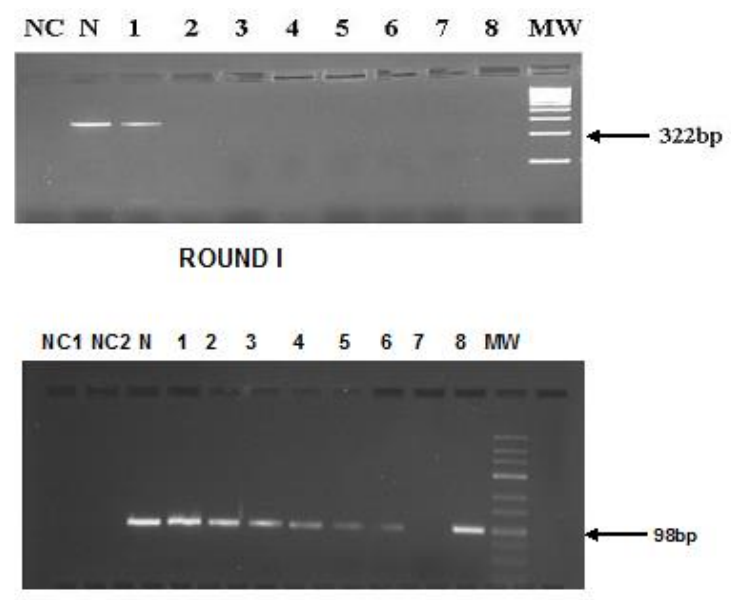

ROUND II

FIGURE 1: Agarose gel electrophoretogram showing sensitivity PCR for LipL32 gene of Leptospira interrogans: Analysis products amplified round $1 \mathrm{NC}$ : Negative control, $\mathrm{N}$ : Neat DNA, Lanes 1-8:10 fold serial dilutions of positive control, DNA (L. interrogans), Mw: Molecular weight marker (100bp).

ROUND 2: NC2: Negative control with round 2 primers, NC1:amplified product of first round negative control transferred from round $1, \mathrm{~N}$ : Neat DNA.

Lanes 1-8:10 fold serial dilutions of DNA extracts of $L$. interrogans, MW: Molecular weight marker (25-700 bp) $98 \mathrm{bp}$ product band was found till $10^{-6}(1 \mathrm{fg})$ dilution.

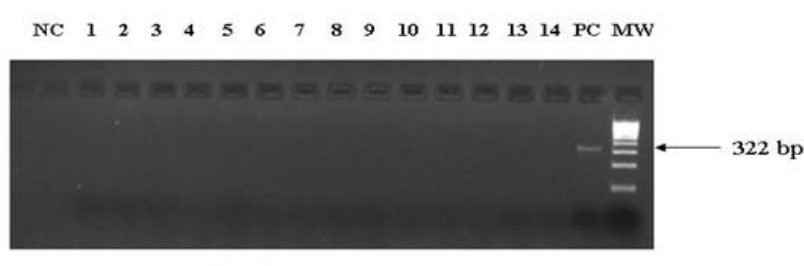

ROUND I

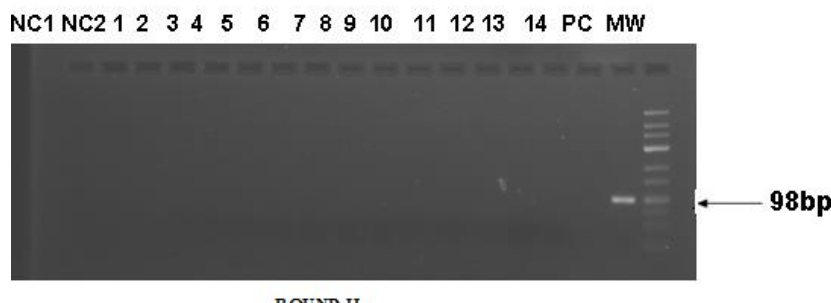

ROUND II

FIGURE 2: Agarose Gel Electrophoretogram Showing Specificity of PCR primers for Lipl32 Gene Of $L$. Interrogans. NC1,NC2 : Negative controls, Lane 1-15:S. aureus ( ATCC 6538), B. subtilis Lab isolate , E.coli (ATCC 4157), Ps.aeroginosa (ATCC 9742), Kleb. pneumoniae lab isolate, M.tuberculosis H37 RV, $P$. vulgaris lab isolate, $H$. influenzae (ATCC16211), Acenitobacter spp (ATCC 9956), S. typhi lab isolate, E.aerogenes lab isolate, C.koseri lab isolate, C.albicans (ATCC 90028), HSV- 1 (ATCC 733-VR), Human DNA, Lane 16: L. interrogans (TANUVAS, Madhavaram, Chennai, India ), MW 17: Molecular weight marker (25700bp).

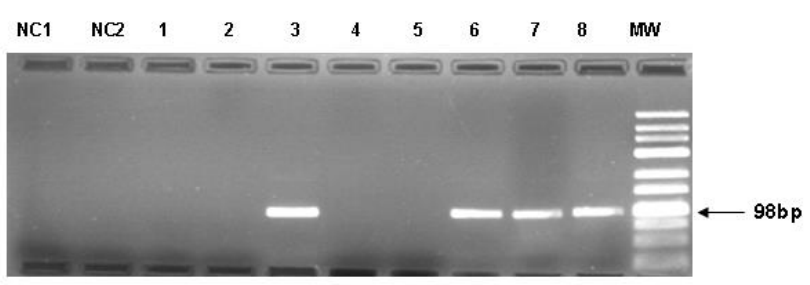

FIGURE 3: Agarose gel electrophoresis showing amplification of LipL32 gene of Leptospira interrogans among culture positive blood samples..: NC1 :first round Negative control, NC2 Second round negative control 2, Lanes: 3,6,7 - DNA extracts from culture positive blood specimens positive for Lip 32 gene Lane 1,2,4,5 - DNA extracts from culture positive blood specimens negative for Lip 32 gene, Lane 8 - Leptospira DNA extracted from standard strain of Leptospira interrogans (TANUVAS, Madhavaram), Lane 9 -Molecular weight marker ( 25 700bp ladder).

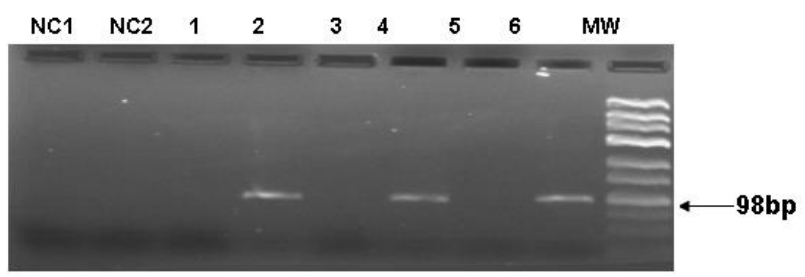

FIGURE 4: Agarose gel electrophoresis showing amplification of LipL32 gene of Leptospira interrogans in culture negative blood samples.

NC1 - First round negative control 1, NC2 Negative control 2, Lane 2,4 - DNA extracts from culture negative blood specimens, but positive for Lip 32 gene, Lane 1, 3, 5- DNA extracts from culture negative blood specimens, negative for Lip 32 gene, Lane 6- Leptospira DNA extracted from standard strain of Leptospira interrogans (TANUVAS, Madhavaram), Lane 7 - Molecular weight marker ( 25-700bp ladder).

For the validation of the primer sensitivity 25 serum samples tested positive by MAT, 25 other samples tested negative for MAT, 20 dark field microscopy positive serum samples and 5 dark field microscopy negative serum samples were collected from Leptospira Diagnostic Centre, TANUVAS, Madhavaram, Tamil Nadu. Serum sample from a patient with neuroleptospirosis was also collected. All these samples were subjected to nPCR targeting 
LipL32 gene. The result showed that primer was sensitive to detected LipL32 gene in all the 25 MAT positive serum samples, 20 dark field microscopy positive serum samples, and the 5 dark field microscopy negative serum samples. The 25 MAT negative serum samples remained negative for PCR. The serum sample from a patient with neuroleptospirosis also turned out to be positive with the nPCR. Thus, the sensitivity of the primer set was determined $100 \%$.

Out of the 207 peripheral blood samples of septicemia patients included in the study 100 samples were positive for bacteria by culture (BacT/ALERT®BIOMERIX). From the remaining 107 samples no microorganisms were isolated. All the 100 culture positive samples were identified using Mini-API system and were further confirmed by PCR using primers specific for each of the bacteria isolated.

PCR targeting LipL32 gene of Leptospira interrogans was applied to all the 207 peripheral blood samples irrespective of the culture reports. Twenty-one out of 100blood culture positive samples (21\%) were found to be positive (Figure 3) for Leptospira interrogans LipL32 gene. Table 1 shows the list of bacteria isolated from 21 positive samples. Among the 107-blood culture negative samples only three $(2.8 \%)$ (Figure 4$)$ showed the presence of Leptospiral DNA (Table 2). Attempts at cultivation of Leptospira from blood samples positive by nPCR failed to isolate the bacterium.

All the PCR positive samples were subjected to DNA sequencing using the forward primer and the sequencing results were analyzed using BLAST software to deduce the sequence homology. Sequences of sixteen out of 21 $(80.9 \%)$ samples had $100 \%$ homology with Leptospira interrogans serovar icterohaemorrhagiae, three out of 21 (23.8\%) showed homology with Leptospira interrogans serovar grippotyphosa and two out of 21 (9.5\%) showed homology with Leptospira interrogans serovar copenhageni. Results for DNA sequencing are shown in (Table 1).

Leptospira was found to be associated with other bacteria in the peripheral blood samples of 24 septicemia patients. The rate of detection of Leptospira among culture positive blood samples was found to significant compared to culture negative samples as shown by significant $p$ value $=<0.001$, (Chi square test).

Brief clinical features among the 24 patients whose peripheral blood samples were positive for Leptospira DNA are as follows. Five patients were initially hospitalized with complaints of brain infarction; their blood culture reports are one each of $S$. aureus, Coagulase negative Staphylococci, Pseudomonas spp, Enterobacter, E. coli. E. coli was isolated in culture from two other patients who had degenerative spinal disease and Parkinson's disease. Five others who had complaints of pyrexia of unknown origin for a period of 5-6 days were positive for Salmonella species by blood culture .Yet another patient with otitis media had $K$. pneumoniae bacteremia, and a patient with fever was blood culture positive for both $S$. aureus and Enterococcus faecalis. Pseudomonas spp was isolated from a patient with community-acquired pneumonia. A 29 year-old female who developed post partum fever due to alpha hemolytic streptococci was also positive for Leptospira. Two had pyrexia of unknown origin in which Staphylococcus aureus was isolated in culture. Eight others were clinically admitted as of pyrexia of unknown origin with blood cultures being positive for bacterium is listed in Table 2 .

\section{DISCUSSION}

Several PCR based methods targeting different locations of Leptospiral genome for specific amplification, hold promise as a molecular tool for the diagnosis of the disease Laboratory diagnosis of Leptospirosis has been an area of interest as it is ill understood. Since conventional culture techniques are cumbersome they are not routinely carried out, Serological tests are preferred, but they have lesser sensitivity. Molecular methods appear to provide definitive diagnosis of this disease (Woo et al., 1999). For designing primers the major outer membrane protein gene LipL32 was chosen as it is proven to be conserved among the pathogenic strains of Leptospira. Van et al., (1989) developed PCR for the detection of Leptospires in urine samples of infected cattle. Gravekamp et al. (1993) proposed the use of two sets of primers (G1 \& G2 and 641 \& B651). However Fonseca et al. (2009) have demonstrated the lesser sensitivity of G1 \& G2 primers in detecting Leptospira from blood samples.

Several lines of evidence indicate that $L i p L 32$ is a lipid modified in a manner similar to that of other prokaryotic lipoproteins. LipL32 is also a prominent immunogen during human Leptospirosis, sequence and expression of LipL32 is highly conserved among pathogenic Leptospira species (David et al., 2000) As this gene is specific to pathogenic Leptospira, detection of pathogenic Leptospira isolates is possible with the nested PCR technique described here. The clinical validation of the primer sets was performed and the primers were specific to amplify all the MAT positive serum samples collected from patients with clinical symptoms of leptospirosis. The five clinical dark ground negative, PCR positive samples were later confirmed MAT positive. A patient with clinical symptoms of neuro leptospirosis, acute renal failure who was MAT positive (titer 1: 200), IgM ELISA (40 PANBIO units) positive and PCR positive, showed immediate recovery from kidney failure and other clinical symptoms after the treatment with interavenous penicillin.

The possible reasons for Leptospira culture failure in all twenty four blood specimens positive for leptospira PCR, the organism could have been inactivated during cold storage period at $-80^{\circ} \mathrm{C}$ as culture was carried out only after the specimens were found positive for leptospira PCR. Since the attempts for the isolation of Leptospira failed, based on the nPCR-based DNA sequencing, we report statistically significant association of pathogenic Leptospira with other bacteria-causing septicemia. As stated earlier Leptospiral co-infection with malarial parasite (Chansuda et al., 2003) dengue virus, (Rele et al., 2001) scrub typhus (Yuhawaiho et al., 2006) and Salmonella (Osbold et al., 2008 and Ronsholt et al., 2009) 
Table 1: Bacteria isolated from the culture positive blood samples nPCR positive but Leptospira was not isolated in culture and DNA sequencing results of 21 blood samples positive for both leptospira PCR and bacteria other than Leptospira by culture.

\begin{tabular}{|c|c|c|c|}
\hline $\begin{array}{l}\text { Specimen } \\
\text { no }\end{array}$ & BACTERIUM ISOLATED & Specimen no & Sequencing results \\
\hline Blood & Klebsiella. pneumoniae & BC 2585/08 & L. interrogans var grippotyphosa \\
\hline culture & Escherichia coli & BC 2606/08 & L. interrogans var grippotyphosa \\
\hline positive & Salmonella typhi & BC 2794/08 & L. interrogans var grippotyphosa \\
\hline \multirow[t]{20}{*}{$\begin{array}{l}\text { specimens } \\
\mathrm{N}=21\end{array}$} & $\begin{array}{l}\text { Escherichia coli / Klebsiella } \\
\text { pneumoniae }\end{array}$ & BC 1260/09 & L. interrogans var copenhageni \\
\hline & Salmonella paratyphi $A$ & BC 1324/09 & L. interrogans var icterohaemorrhagiae \\
\hline & $\begin{array}{l}\text { Methicillin Resistant } \\
\text { Staphylococcus aureus }\end{array}$ & BC 1326/09 & L interrogans var .icterohaemorrhagiae \\
\hline & Escherichia coli & BC 1327/09 & L. interrogans var icterohaemorrhagiae \\
\hline & Salmonella typhi & BC 1423/09 & L. interrogans var icterohaemorrhagiae \\
\hline & Salmonella typhi & BC 1651/09 & L. interrogans var copenhageni \\
\hline & Enterobacter aerogenes & BC 1655/09 & L. interrogans var icterohaemorrhagiae \\
\hline & Salmonella typhi & BC 1662/09 & L. interrogans var icterohaemorrhagiae \\
\hline & Staphylococcus aureus & BC 1672/09 & L. interrogans var icterohaemorrhagiae \\
\hline & E. faecalis & BC 1689/09 & L. interrogans var icterohaemorrhagiae \\
\hline & Alpha hemolytic Streptococci & BC 1693/09 & L. interrogans var icterohaemorrhagiae \\
\hline & $\begin{array}{l}\text { Staphylococcus aureus / } \\
\text { Enterococcus faecalis }\end{array}$ & BC 1738/09 & L. interrogans var icterohaemorrhagiae \\
\hline & Pseudomonas aeuroginosa & BC 1805/09 & L. interrogans var icterohaemorrhagiae \\
\hline & Coagulase Negative & BC 1843/09 & L. interrogans var icterohaemorrhagiae \\
\hline & Staphylococcus aureus & & \\
\hline & Escherichia coli & BC 1857/09 & L. interrogans var icterohaemorrhagiae \\
\hline & Coagulase Negative & BC 1864/09 & L. interrogans var icterohaemorrhagiae \\
\hline & Staphylococcus aureus & & \\
\hline & Klebsiella. pneumoniae & BC 1916/09 & L. interrogans var icterohaemorrhagiae \\
\hline & Pseudomonas aeuroginosa & BC 1935/09 & L. interrogans var icterohaemorrhagiae \\
\hline \multirow{3}{*}{\multicolumn{2}{|c|}{$\begin{array}{l}\text { Blood culture Negative Specimens } \\
\mathrm{N}=3\end{array}$}} & BC 2841/09 & L. interrogans var grippotyphosa \\
\hline & & BC 2844/09 & L. interrogans var grippotyphosa \\
\hline & & BC 1815/09 & L. interrogans var icterohaemorrhagiae \\
\hline
\end{tabular}

Note: Table 1 shows the list of bacteria isolated in culture by automated BacT/ALERT®BIOMERIX from the 21 culture positive blood samples which were also positive for Leptospira interrogans by nested PCR targeting LipL32 gene.Among the 207 clinically suspected cases of septicemia 21samples which were positive for bacteria by blood culture and 3 among the culture negative groups showed the presence of Leptospira LipL32 gene. Further sequencing of PCR amplified products showed homology with Leptospira interrogans, DNA sequencing, the results are given in the following table.

Table 2: PCR results of LipL32 gene of Leptospira interrogans on both culture positive and culture negative blood samples

\begin{tabular}{cccccc}
\hline S.NO & $\begin{array}{c}\text { Specimen details } \\
\mathrm{N}=207\end{array}$ & Total number & \multicolumn{2}{c}{ nPCR for LipL32 gene } & Percentage of \\
\cline { 3 - 4 } & & & POSITIVE & NEGATIVE & positivity \\
\hline 1 & Blood culture positive specimens & 100 & 21 & 79 & $21 \%$ \\
2 & Blood culture Negative Specimens & 107 & 3 & 104 & $2.8 \%$ \\
\hline
\end{tabular}

have been reported in the previous studies. Chansuda et al. (2003) reported the association of Leptospira with malaria in two patients mainly by demonstration of MAT. Our observation provides compelling evidence for coinfection of Leptospira in the form of its presence as DNA with other bacteria-causing septicemia. Based on this significance of the data, it is hypothesized that there exists an increased susceptibility of individuals with bacteremia to Leptospira infection. Most of these associations were found with Gram-negative bacteria especially organisms belonging to the family. The 24 Leptospira nPCR positive samples were subjected to DNA sequencing, and they were identified as Leptospira interrogans var icterohaemorrhagiae followed by Leptospira interrogans var grippootyphos though the DNA sequenced was only $98 \mathrm{bp}$ in length, the blast result showed $98 \%$ homology (http://last.ncbi.nlm.nih.gov/blast.cgi).

In our study, we observed that patients who were hospitalized for brain infarction, subsequently developed nosocomial urinary tract infections and showed positivity for blood culture predominantly with Enterobacteriaceae group of bacteria, were highly prone to Leptospiral co- 
infection, which probably have not been thought of clinically. Followed by them were patients who had pyrexia of unknown origin from whom Salmonella spp. was isolated in culture. As mentioned earlier, Leptospira can remain in immunologically privileged sites especially renal tubules after clearance from blood and can persist for longer than 60 days. It is our hypothesis that patients with primary bacterial infection may have decreased immune status could have developed Leptospiral coinfection. The source of Leptospira infection in these patients is probably endogenous. The bacterium remaining in the renal tubules might have seeded into the blood stream due to immuno suppression. In the three patients who were Leptospira nPCR positive and blood culture negative, may either have had only leptospirosis. Since PCR is the most sensitive method, leptospirosis was excluded in those samples that failed to amplify LipL32 gene.

It is accepted that the use of culture confirms diagnosis but is not generally used since it is rarely successful and has low sensitivity. In this study the cultures for Leptospira were attempted only in PCR positive samples, which unfortunately were stored frozen before culture was attempted. Moreover, these patients were on antibiotics at the time of admission and these might have might have acted on Leptospira for a period of time even before blood was collected and also during storage period. It may be noted the successful isolation of other bacteria was done directly into blood culture media (performed immediately after blood sample was collected from patient) where the antibiotics would have been either diluted or inactivated.

In this study an nPCR was successfully developed and optimized using standard strains and was applied on consecutive 207 blood cultures being performed on patients with clinical diagnosis of PUO or Septicaemia. Wherever Leptospira DNA was detected the PCR product was sequenced to its identity and was proven to be Leptospira interrogans. When the results were further analyzed in relation to the results of bacterial cultures, to our surprise, statistically significant number of bacterial culture positive blood specimens showed the presence of leptospira DNA. This surprise finding made us to hypothesize that an increased susceptibility of the individual with bacteremia to leptospira infection which probably was due to an activated disease of an endogenous origin. This well documented new information with PCR-based DNA sequencing had proven them to be the pathogenic species of Leptospira; it might be of great importance in the pathogenesis of leptospirosis.

Moreover, the lack of correlation between clinical symptoms and laboratory diagnosis is already well described in the literature (Angnani et al., 2001 and Katz et al., 2001). Apart from the above reasons, other workers have clearly stated that human leptospirosis is not commonly diagnosed in most of these places as it may have been under reported due to lack of awareness; its protean clinical manifestations and non-availability of the laboratory tests (Ahmad et al., 2005). The MAT is currently considered to be the reference serologic test for leptospirosis infection, but the lack of standardization of base line titers in the community influences test validity and may result in overdiagnosis and overestimation of disease burden. Due to the antigenic heterogeneity of Leptospira species the MAT test requires many serovars as antigens. As the designed nPCR showed positivity for the respective MAT positive serum samples and negative results to the respective MAT negative serum samples the method appears to be more sensitive, specific and is most reliable. Polymerase chain reaction gains advantage over MAT as early stages of infection can be detected. Overall, we conclude that nPCR standardized with primers targeted to LipL32 is highly sensitive, specific and reliable for detection of leptospira in peripheral blood.

\section{Acknowledgement}

The author acknowledges Vision Research Foundation for the funding.

\section{REFERENCES}

Ahmad, S. N., Shah, S., Ahmad, F. M. H. (2005). Laboratory diagnosis of leptospirosis. Journal of Post Graduate Medicine 51, 195-200.

Agampodi, S. B., Peacock, S. J., Thevanesam, V., Nugegoda, D. B., Vinetz, J. M. (2011). Leptospirosis Outbreak in Sri Lanka in 2008: Lessons for Assessing the Global Burden of Disease. Journal of Tropical Medicine Hygiene 85 (3), 471-478.

Angnani, R., Pathak, A. A., Mishra, M. (2001). Prevalence of Leptospirosis in various risk groups. Indian Journal of Medical Microbiology 21, 271-273.

Center for Disease Control and Prevention (1998). Outbreak of acute febrile illness among athletes participating in triathlons-Wisconsin and Illinois. Morbidity and Mortality Weekly Report 47, 585-588.

Center for Disease Control and Prevention (2000). Leptospirosis outbreak in Eco Challenge 2000 participants. Communicable Disease Report Weekly 10, 341.

Chansuda, W., Clinton, K., Miller, R., Philip Mc Daniel, Wilson, J., Liao et al. (2003). Co-infection with Malaria and Leptospirosis. American Journal of Tropical Medicine and Hygiene 68 (5), 583-585.

Chu, K. M., Rathinam, R., Namperumalsamy, P., Dean, D. (1998). Identification of Leptospira species in the pathogenesis of uveitis and determination of clinical ocular characteristics in South India. Journal of Infectious Diseases 177, 1314-1321.

David, A. H., Chao, G., Zuerner, R. L., Jeanne, K. B., Dean, B., Mary, M., James, M., Paul, N. L., Carole, A. B. (2000) .The Leptospiral Major Outer Membrane Protein LipL32 Is a Lipoprotein Expressed during Mammalian Infection. Infection and Immunity 68 (4) 2276-2285.

Dey, S., Madhan Mohan, C., Ramadass, P., Nachimuthu ,K. (2007). Recombinant antigenbased latex agglutination test for rapid serodiagnosis 
of Leptospirosis. Veterinary Research Communications 31 (1), 9-15.

Faine, S. (1994). Leptospira and Leptospirosis. In: Taxonomy, classification and Nomenclature. CRC press, London 117-140.

Fonseca, C., Teixeira, M., Romero, E., Tengan, F., Silva, M., Shikanai Yasuda, M. (2009). Leptospira DNA detection for the diagnosis of human leptospirosis. Journal of Infection 52 (1), 15-22.

Gravekamp, H., Van de Kemp, M., Franzen, D., Carrington, G. J., Schoone, G. J. J. M., Van Eys et al. (1993). Detection of seven species of pathogenic leptospires by PCR using two sets of primers. Journal of General Microbiology 139, 1691-1700.

Haake, D. A., Suchard, M. A., Kelly, M. M., Dundoo, M., Alt, D. P., Zuerner, R. L. (2004). Molecular evolution and mosaicism of Leptospiral outer membrane proteins involves horizontal DNA transfer. Journal of Bacteriolology 9, 2818-2828.

Hernandez, P. et al. (2011). A comparison between polymerase chain reaction (PCR) and traditional techniques for the diagnosis of leptospirosis in bovines. Journal of Microbiological Methods 84, 1-7.

Jena, A. B., Mohanty, K. C., Devadasan, N. (2004). An outbreak of leptospirosis in Orissa, India: The importance of surveillance. Tropical Medicine and International Health 9, 1016-1021.

Jyoti, S., Urmil, T. and Harsh, V. B. (2004). DNA probes for the identification of Leptospires and disease diagnosis. Southeast Asian Journal of Tropical Medicine and Public Health 35(2), 346-352.

Katz, A. R., Ansdell, V. E., Effler, P. V., Middleton, C. R., Sasaki, D. M. (2001). Assessment of the Clinical Presentation and Treatment of 353 Cases of Laboratory-Confirmed Leptospirosis in Hawaii, 19741998. Clinical Infectious Diseases 33, 1834-1841.

Marianelli et al. (2007). Molecular detection of Leptospira species in aborted fetuses of water buffalo. Veterinary Record 161, 310-311.

Mérien, P., Amouriaux, P., Perolat, G., Baranton and Saint, G. (1992). Polymerase chain reaction for detection of Leptospira spp. in clinical specimens. Journal of Clinical Microbiology 30 (9), 2219-2224.

Noubade, R., Krishnamurthy, G., Murag, S., Venkatesha, M., Krishnappa, G. (2002). Differentiation of pathogenic and saprophytic leptospires by polymerase chain reaction. Indian Journal of Medical Microbiology 20 (1), 33-36.

Osebold, William, R. (2008). Systemic Leptospirosis Followed by Salmonella Vertebral Osteomyelitis Without Sickling or Immunosuppression. Spine 33 (2), 55-61.

Pan American Health Organization. (1998). Impact of Hurricane Mitch in Central America. Boletin Epidemiologico 19, 1-13.
Paul, N., Levett, Roger, E., Morey, Renee, L., Galloway, Danielle, E., Turner, Arnold, G., Steigerwalt and Leonard, W., Mayer. (2005). Detection of pathogenic Leptospires by real-time quantitative PCR. Journal of Medical Microbiology 54, 45-49.

Rele, M. C., Rasal, A., Despande, S. D., Koppikar, G. V,. Lahiri, K. R. (2001). Mixed infection due to Leptospira and Dengue in a Patient with pyrexia. Indian Journal of Medical Microbiology 19 (4), 206207.

Rönsholt, F. F., Seidelin, J. B., Villumsen, S. (2009). Concurrent leptospirosis and salmonella infection. Ugeskr laeger 171 (19), 1607-1609.

Suganthan, S., Varghese, T. P. (2005). Multiplex PCR on Leptospiral isolates from Kolenchery, Kerala, India. Indian Journal of Medical Microbiology 23 (2), 114116.

Sanders, E. J., Rigau-Perez, J. G., Smits, H. L., Deseda, C. C., Vorndam, V. A., Aye, T., Spiegel, R. A. et al. (1999). Increase of Leptospirosis in denguenegative patients after a hurricane in Puerto Rico in 1996. American Journal of Tropical Medicine and Hygine 61, 399-404.

Suganthan, S. Varghese, T.P. (2005). Multiplex PCR on Leptospiral isolates from Kolenchery, Kerala, India. Indian Journal of Medical Microbiology 23 (2), 114116.

Van Eus, G. K. K., Gravekemp, C., Gerritsen, M. J., Quint, W., Cornelissen, M. T. E. et al. (1989). Detection of Leptospires in urine by the polymerase chain reaction Journal of Clinical Microbiology 27, 2258-2262.

Woo ,T. H. S., Patel, B. K. C., Symonds, M. L., Norris, M. A., Dohnt, M. R. (1999). Comparison of two PCR methods for rapid Leptospira genome species interrogans. FEMS Microbiology Letters 155, 169 177.

Wuthiekanun, V., Sirisukkarn, N., Daengsupa, P., Sakaraserane, P., Sangkakam, N., Chierakul, W., Smythe, L. D., Symonds, M. L., Dohnt, M. F., Slack, A. T., Day, N. P., Peacock, S. J. (2007). Clinical Diagnosis and Geographic Distribution of Leptospirosis, Thailand. Emerging Infectious Diseases 13, 124-126.

Yu- Huai, H., Li-Kuang, C., Pei-Jane, T., Lih-Shinn, W. (2006). Coinfection with Leptospirosis and Scrub Typhus - A Report of Four Cases. Tzu Chi Medical Journal 18 (23). 